\title{
Master Schedule for CY-1977 Hanford Environmental Surveillance Routine Program
}

\author{
by \\ P. J. Blumer \\ D. A. Myers \\ J. J. Fix
}

December 1976

Prepared for the Energy Research and Development Administration under Contract E(45-1)-1830 
NOTICE

This report was prepared as an account of work sponsored by the United States Government. Neither the United States nor the Energy Research and Development Administration, nor any of their employees, nor any of their contractors, subcontractors, or their employees, makes any warranty, express or implied, or assumes any legal liability or responsibility for the accuracy, completeness or usefulness of any information, apparatus, product or process disclosed, or represents that its use would not infringe privately owned rights.

\title{
PACIFIC NORTHWEST LABORATORY
}

operated by

BATTELLE

for the

ENERGY RESEARCH AND DEVELOPMENT ADMINISTRATION

Under Contract EY-76-C-06-1830

\author{
Printed in the United States of America \\ Available from \\ National Technical Information Service \\ U.S. Department of Commerce \\ 5285 Port Royal Road \\ Springfield, Virginia 22151
}

Price: Printed Copy \$_ *; Microfiche $\$ 3.00$

NTIS

- Pages Selling Price

$001-025 \quad \$ 4.50$

026-050 $\$ \$ 5.00$

051-075 $\$ 5.50$

076-100 $\$ 6.00$

$101-125 \quad \$ 6.50$

$126-150 \quad \$ 7.00$

$\begin{array}{ll}151-175 & \$ 7.75\end{array}$

$176-200 \quad \$ 8.50$

$201-225 \quad \$ 8.75$

$226-250 \quad \$ 9.00$

$251-275 \quad \$ 10.00$

$276-300 \quad \$ 10.25$ 
33679000627069

MASTER SCHEDULE FOR CY-1977

HANFORD ENV IRONMENTAL SURVEILLANCE

ROUTINE PROGRAM

\author{
by \\ P. J. Blumer \\ D. A. Myers \\ J. J. Fix
}

December 1976

BATTELLE

Pacific Northwest Laboratories

Richland, Washington 99352 


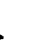

. 


\section{CONTENTS}

INTRODUCTION

SCHEDULE

I. AIR

II. COLUMBIA RIVER

III. SANITARY WATER

IV. SURFACE WATER

V. GROUNDWATER

200 Area Wells

600 Area Wells

100 Area Wells

300 Area We11s

Quality of Water Analyses Annually $\quad$. . . . . . . $\quad$. 14

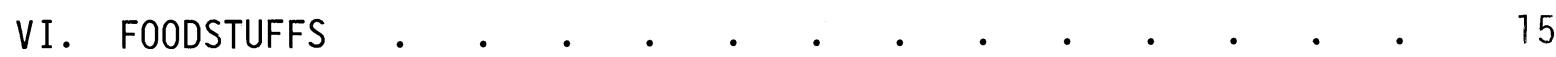

$\operatorname{Milk}$. . . . . . . . . . . . . . . 15

Meat . . . . . . . . . . . . . . 15

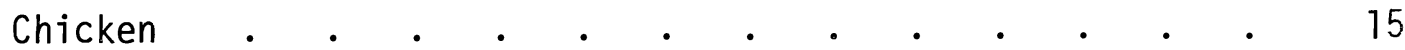

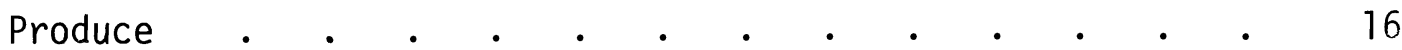

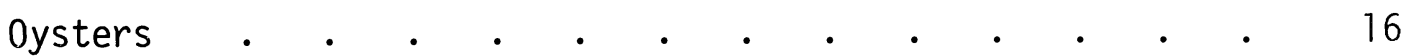

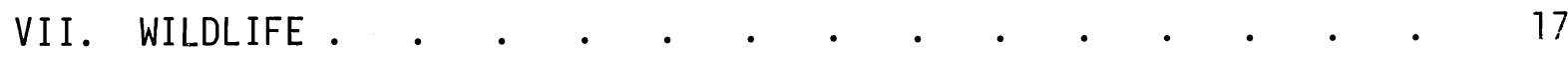

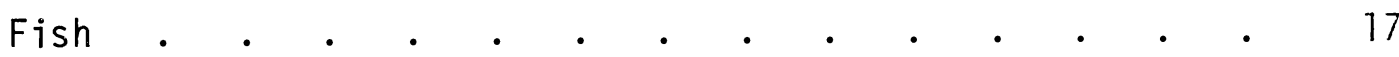

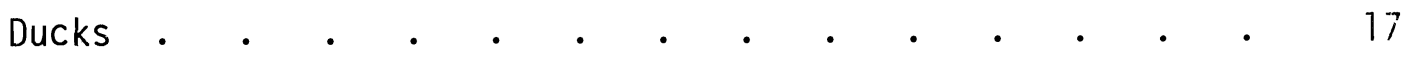

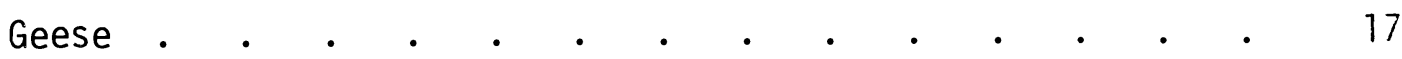

Pheasants • . . . . . . . . . . . . . 17

Deer . . . . . . . . . . . . . . . 17

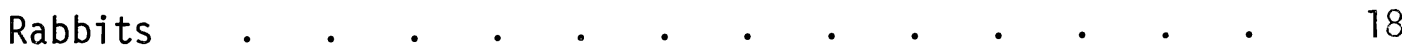

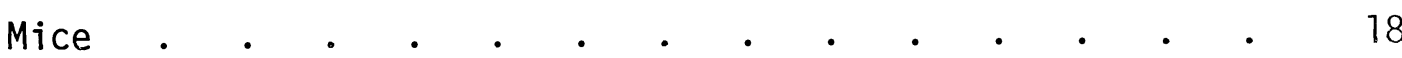

Other Smal1 Mammals . . . . . . . . . . . . . 18

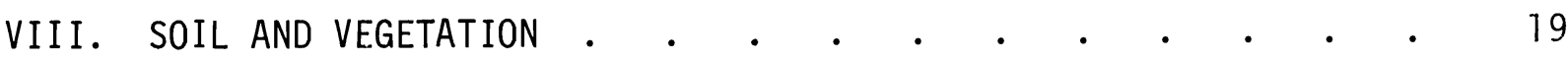

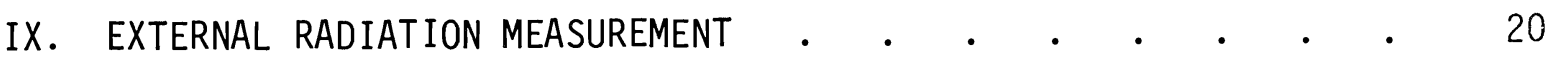

Thermoluminescent Dosimeters . . . . . . . . 20 


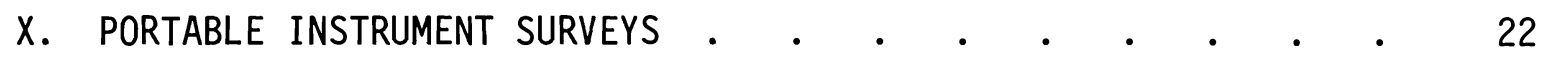

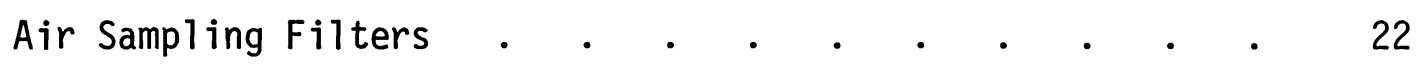

Columbia River Shoreline . . . . . . . . . . 22

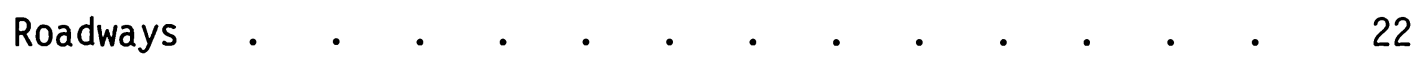

Railroad Survey . . . . . . . . . . .

Aerial Survey . . . . . . . . . . . . . 24

XI. SURVEILLANCE OF WASTE DISPOSAL SITES • • • • • • • . 25

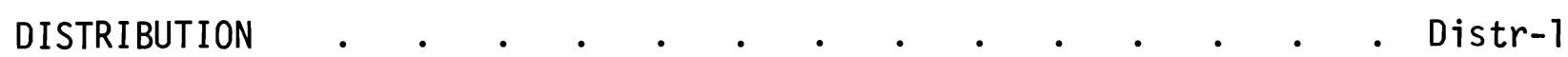


MASTER SCHEDULE FOR CY-1977

HANFORD ENVIRONMENTAL SURVEILLANCE ROUTINE PROGRAM

\title{
INTRODUCTION
}

This report provides the current schedule of data collection for the routine environmental surveillance program at the Hanford Site, as conducted by the Environmental Evaluation Section of Battelle, Pacific Northwest Laboratory for the Energy Research and Development Administration (ERDA). Questions about specific entries should be referred to the authors since modifications to the schedule are made during the year and special areas of study, usually of short duration, are not scheduled.

The environmental surveillance program objectives are to evaluate the levels of radioactive and nonradioactive pollutants in the Hanford environs, as required in ERDAM-0513, and to monitor Hanford operations for compliance with applicable environmental criteria given in ERDAM-0524 and Washington State Water Quality Standards. Air quality data are obtained in a separate program administered by the Hanford Environmental Health Foundation.

The data collected are available in routine reports issued by the Environmental Evaluations staff. Groundwater data and evaluation are reported in the series, "Radiological Status of the Groundwater Beneath the Hanford Project for ...," the latest issue being BNWL-2034 for CY-1975. Data from locations within the plant boundaries are presented in the annual "Environmental Status of the Hanford Reservation for ..." report series, the most recent report being BNWL-B-477 for 1975. Data from offsite locations are presented in the annual "Environmental Surveillance at Hanford for ..." series of reports, the latest being BNWL-1979 for 1975 .

\author{
Frequency Symbols Used

$\begin{array}{rr}\text { D - Daily } & \text { M Comp. - Monthly Composite } \\ \text { W - Weekly } & \text { - Quarterly } \\ \text { BW - Biweekly } & \text { A - Annually } \\ \text { M - Monthly } & \text { SA - Semi-annually } \\ \text { BM - Bimonthly } & \text { NRA - Not Routinely Analyzed }\end{array}$


I. $\underline{A I R}$

Location

Onsite

$100-K$

100-N (WPPSS)

100-D

100-F

100-A Fire Station

200 WEC

200 WWC

Redox

200 ESE

200 ENC

200 WNE

200 EEC

200 EWC

Hanford

Wye Barricade

FFTF

3705 Bldg.

ACRMS

300 SW Gate

300 South Gate

300 Pond

RRC CP\#63

RRC CP\#64

Prosser Barr.

ERC

Rattlesnake Spr.

Yakima Barr.

Vernita Bridge

Wahluke Slope \#2

Offsite

Berg Rance

Othello

Connel1

Cooke Bros.

Wahluke Wm.

Baxter Substation

Byers Landing

Pasco

Richland

Benton City

Moses Lake

Washtucna

Walla Walla

McNary Dam

Sunnyside
EMA

1529

1074

1075

6154

0046

0045

0028

0043

1401

1370

0042

0041

0057

0924

6308

1531

1793

6148

6150

1543

6180

6182

0055

0929

0972

1650

1651

1551

1405

1652

1653

1118

1119

6235

0247

1654

0054

0029

0960

0959

0262

0958

0964
Filter

Frequency Analyses

Beta

Beta

Beta

Beta

Beta

Beta, Lo Alpha

Beta

Beta, Lo Alpha

Beta, Lo Alpha

Beta, Lo Alpha

Beta

Beta, Lo Alpha

Beta

Beta

Beta, Lo Alpha

Beta, Lo Alpha

Beta

Beta

Beta

Beta, Lo Alpha

Beta

Beta, Lo Alpha

Beta

Beta, Lo Alpha

Beta

Beta

Beta

Beta

Beta

Beta, Lo Alpha

Beta

Beta

Beta

Beta

Beta

Beta, Lo Alpha

Beta

Beta, Lo Alpha

Beta, Lo Alpha

Beta

Beta

Beta

Beta

Beta
Composite ${ }^{(a)}$

Group

100 Areas

100 Areas

100 Areas

100 Areas

100 Areas

200 West

200 West

200 West

200 East

200 East

200 West

200 East

200 East

IE

IE

IE

300 Area

300 Area

300 Area

300 Area

300 Area

ISE

ISE

ISW

ISW

ISW

INW

INW

INW

INE

INE

INE

INE

INE

ISE

ISE

ISE

ISE

ISW

ONE

ONE

OSE

OSE

OW

(a) Composite group for filter. Analyses shown on Page 4 . 


\section{AIR (continued)}

Location

Onsite

$100-K$

$100-\mathrm{N}$ (WPPSS)

$100-D$

$100-\mathrm{F}$

100-A Fire Station

200 WEC

200 WWC

Redox

200 ESE

200 ENC

200 WNE

200 EEC

200 EWC

Hanford

Wye Barricade

FFTF

3705 B1dg.

ACRMS

300 SW Gate

300 South Gate

300 Pond

RRC CP\#63

RRC CP\#64

Prosser Barr.

ERC

Rattlesnake Spr.

Yakima Barr.

Vernita Bridge

Wahluke Slope \#2

Offsite

Berg Ranch
0 thello
Connell
Cooke Bros.
Wahluke Wm.
Baxter Substation
Byers Landing
Pasco
Richland
Benton City
Moses Lake
Washtucna
Walla Walla
McNary Dam
Sunnyside

Charcoal Cartridge

EMA \# Frequency Analyses EMA \# Frequency Analyses

1581

1661

1582

1583

6155

1662

6152

1663

1664

1665

6272

1361

6273

1666

1584

6309

1669

1795

6149

6151

6239

6181

6183

6248

1585

1586

1667

1668

1671

$\begin{array}{ll}M & \text { NRA } \\ M & \text { NRA } \\ \text { BW } & I-131\end{array}$

M NRA

$M \quad$ NRA

$M \quad$ NRA

$M \quad$ NRA

M NRA

$\begin{array}{lllll}\text { BW } & \text { I-131 } 6201 & \text { BW } & \text { HTO }\end{array}$

M NRA

$M \quad$ NRA

$M \quad$ NRA

$M \quad$ NRA

$M \quad$ NRA

$M \quad$ NRA

$M \quad$ NRA

$M \quad$ NRA

$M \quad$ NRA

BW I-131

M NRA

$M \quad$ NRA

M NRA

$M \quad$ NRA

$M \quad$ NRA

$M \quad$ NRA

$M \quad$ NRA

$M \quad$ NRA

$M \quad$ NRA

M NRA

1672

1673

1674

1675

1676

6236

0246

1678

0231

1670

1682

1683

0261

1684

1680

$M$

Tritium Cartridge

BW

HTO

6335

HTO

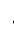


I. AIR (continued)

\section{Composite Group}

200 East 200 ENC

200 EWC

200 ESE

200 EEC

\section{West \\ 200 WEC \\ 200 WWC \\ Redox}

200 WNE

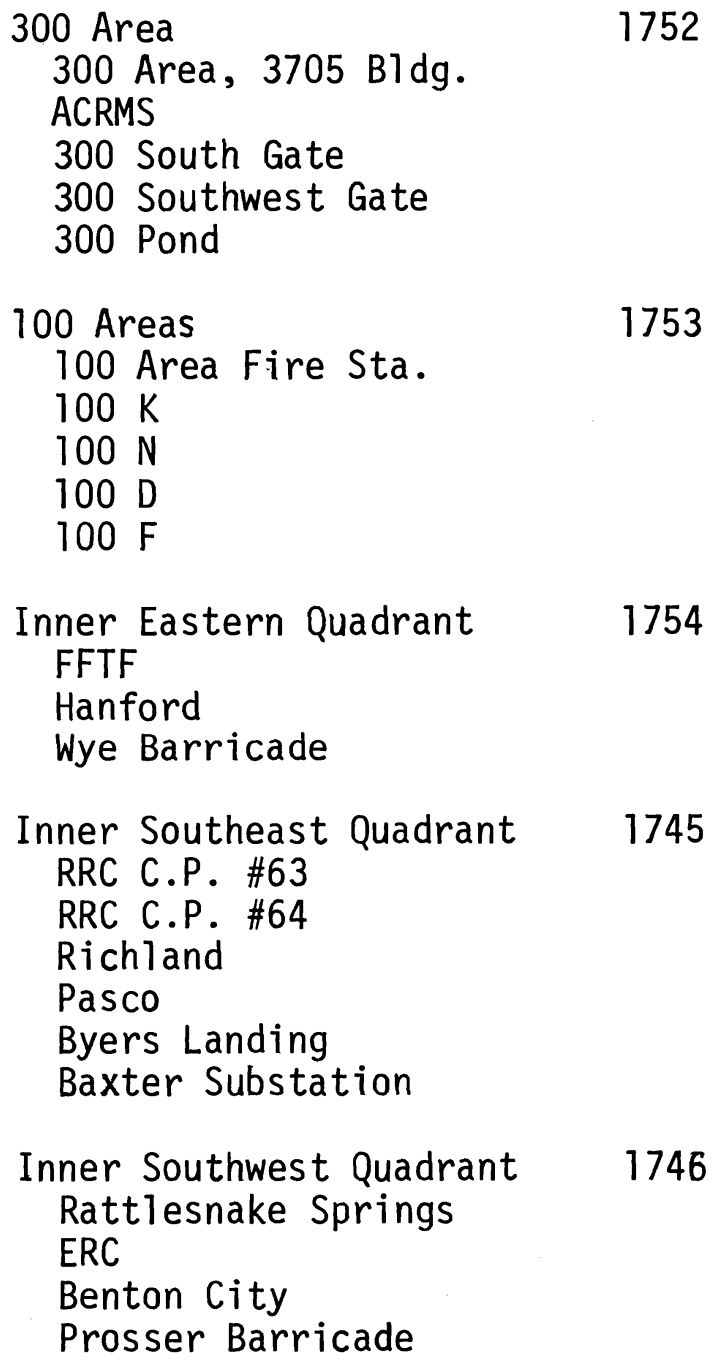

1752

1753

1754

1745

1746

EMA \#

1749

1751
M

Q

$M$

Q

M

Q
M

Q

Frequency

M

Q

M

Q

Gamma scan

Sr-90, Pu-total

Gamma scan

Sr-90, Pu-total
Gamma scan

Sr-90, Pu-tota 1
Gamma scan

Sr-90, Pu-total

Gamma scan

Sr-90, Pu-total
Gamma scan

Sr-90, Pu-total 
I. AIR (continued)

\begin{tabular}{|c|c|c|c|}
\hline Composite Group & EMA \# & Frequency & Analyses \\
\hline $\begin{array}{l}\text { Inner Northwest Quadrant } \\
\text { Yakima Barricade } \\
\text { Vernita } \\
\text { Wahluke \#2 }\end{array}$ & 1747 & $\begin{array}{l}M \\
Q\end{array}$ & $\begin{array}{l}\text { Gamma scan } \\
\text { Sr-90, Pu-total }\end{array}$ \\
\hline $\begin{array}{l}\text { Inner Northeast Quadrant } \\
\text { Othel 10 } \\
\text { Connel1 } \\
\text { Berg Ranch } \\
\text { Wahluke Watermaster } \\
\text { Cooke Bros. }\end{array}$ & 1743 & $\begin{array}{l}M \\
Q\end{array}$ & $\begin{array}{l}\text { Gamma scan } \\
\text { Sr-90, Pu-total }\end{array}$ \\
\hline $\begin{array}{l}\text { Outer Northeast Quadrant } \\
\text { Moses Lake } \\
\text { Washtucna }\end{array}$ & 1742 & $\begin{array}{l}M \\
Q\end{array}$ & $\begin{array}{l}\text { Gamma scan } \\
\text { Sr-90, Pu-total }\end{array}$ \\
\hline $\begin{array}{l}\text { Outer Southeast Quadrant } \\
\text { Walla Walla } \\
\text { McNary }\end{array}$ & 1744 & $\begin{array}{l}M \\
Q\end{array}$ & $\begin{array}{l}\text { Gamma scan } \\
\text { Sr-90, Pu-total }\end{array}$ \\
\hline $\begin{array}{l}\text { Outer Western Quadrant } \\
\text { Sunnyside }\end{array}$ & 1748 & $\begin{array}{l}M \\
Q\end{array}$ & $\begin{array}{l}\text { Gamma Scan } \\
\text { Sr-90, Pu-total }\end{array}$ \\
\hline
\end{tabular}


II. COLUMBIA RIVER

Sample Type

"Filter-Resin"

Priest Rapids Dam

300 Area Forebay

Cumulative $\quad 100-\mathrm{B}$

$\underline{G r a b}$

Vernita

Richland

1000

6156

$Q$

$\mathrm{BW}$
$\mathrm{M}$
$\mathrm{Q}$

BW

Q

M comp

M comp

Q

1204

1616

1373 *

1615

Hanford Powerline

6208

300 Area Forebay

6134

Richland Forebay

1041

1617

1365
W

W

$M^{*}$

M comp

M

W

$M^{*}$

M Comp

W

$M^{*}$
Analyses

Gamma Scan ( ${ }^{131} \mathrm{I}$ )

${ }^{129} \mathrm{I}$

${ }^{90} \mathrm{Sr}, \mathrm{Pu}$

Gamma Scan ( ${ }^{31} \mathrm{I}$ )

$129 \mathrm{I}$

${ }^{90} \mathrm{sr}, \mathrm{Pu}$

Alpha, Beta, ${ }^{3} \mathrm{H}$,
Gamma Scan, $U$
${ }^{89} \mathrm{Sr},{ }^{\circ} \mathrm{Sr}, 226 \mathrm{Ra}$
$228 \mathrm{Ra}$

Alpha, Beta

${ }^{3} \mathrm{H}$, Gamma Scan, U

${ }^{89} \mathrm{Sr},{ }^{0} \mathrm{Sr},{ }^{226} \mathrm{Ra}$, $228 \mathrm{Ra}$

pH, Diss $0_{2}$, Turbidity

$\mathrm{NO}_{3}$

Coliform, Fecal

Coliforms, BOD,

${ }^{3} \mathrm{H}$

Alpha, Beta

$\mathrm{pH}$, Diss $\mathrm{O}_{2}$, Turbidity

Col iform, BOD, Fecal Coliforms

${ }^{3} \mathrm{H}$

$\mathrm{NO}_{\overline{3}}$

Coliform, BOD, Fecal Coliforms

* Performed by HEHF 
III. SANITARY WATER

\begin{tabular}{|c|c|c|c|c|}
\hline Sample Type & Locations & EMA \# & Frequency & Analyses \\
\hline \multirow[t]{5}{*}{ Cumulative } & $181-N$ & 1367 & $\begin{array}{l}\text { W } \\
M \text { Comp }\end{array}$ & $\begin{array}{l}\text { Beta } \\
\text { Gamma Scan }\end{array}$ \\
\hline & 300 Area (River) & $\begin{array}{l}1686 \\
1724\end{array}$ & $\begin{array}{l}W \\
W *\end{array}$ & $\begin{array}{l}\text { Alpha, Beta } \\
\mathrm{NO}_{3}^{-}\end{array}$ \\
\hline & $\begin{array}{r}309 \text { We11 (a) } \\
(399-4-5)\end{array}$ & $\begin{array}{l}6300 \\
6315\end{array}$ & $\begin{array}{l}W \\
M \text { Comp } \\
W^{*}\end{array}$ & $\begin{array}{l}\text { Alpha, Beta } \\
\text { Gamma Scan, }{ }^{3} \mathrm{H}, \mathrm{U} \\
\text { NO} \overline{3}\end{array}$ \\
\hline & Richland & 1002 & $\begin{array}{l}\text { W } \\
\text { M Comp }\end{array}$ & $\begin{array}{l}\text { Alpha, Beta } \\
\text { Gamma Scan }\end{array}$ \\
\hline & & 1366 & $W^{*}$ & $\mathrm{NO}_{3}^{-}$ \\
\hline \multirow[t]{4}{*}{ Grab } & FFTF & 6301 & $\begin{array}{l}M \\
Q\end{array}$ & $\begin{array}{l}\text { Beta, Alpha } \\
3 \mathrm{H}, \text { Gamma Scan } \\
{ }^{89} \mathrm{Sr},{ }^{90} \mathrm{Sr}, \\
226 \mathrm{Ra},{ }^{228} \mathrm{Ra}\end{array}$ \\
\hline & WPPSS & 6297 & $\begin{array}{l}M \\
Q\end{array}$ & $\begin{array}{l}\text { Beta, Alpha, } \\
{ }^{3} \mathrm{H}, \text { Gamma Scan } \\
{ }^{89} \mathrm{Sr},{ }^{90} \mathrm{Sr}, \\
{ }^{2}{ }^{6} \mathrm{Ra},{ }^{228} \mathrm{Ra}\end{array}$ \\
\hline & ALE Hdqtrs & 6299 & M & $\begin{array}{l}\text { Alpha, Beta, }{ }^{3} \mathrm{H} \\
\text { Gamma Scan }\end{array}$ \\
\hline & & & Q & $\begin{array}{l}{ }^{89} \mathrm{Sr},{ }^{90} \mathrm{Sr}, \\
226 \mathrm{Ra},{ }^{228} \mathrm{Ra}\end{array}$ \\
\hline
\end{tabular}

(a) Collected only upon notification by HEDL that the well is being used. * Performed by HEHF 
IV. SURFACE WATER

Location

Gable Mountain Pond

West Lake

B Pond

T Pond

U Pond

Ditch \#216-B-63

$\frac{\text { EMA \# }}{1054} \quad \frac{\text { Frequency }}{Q}$

6133

0015

1290

0024

1725
$Q$

Q

Q

Q

Q
Analyses

Alpha, Beta, Gamma Scan, $90 \mathrm{Sr}$

Alpha, Beta, Gamma Scan, 90 Sr

Alpha, Beta, Gamma Scan, 90 Sr

Alpha, Beta, Gamma Scan, $90 \mathrm{Sr}$

Alpha, Beta, Gamma Scan, $90 \mathrm{Sr}$

Alpha, Beta, Gamma Scan 
V. GROUNDWATER

Analyses

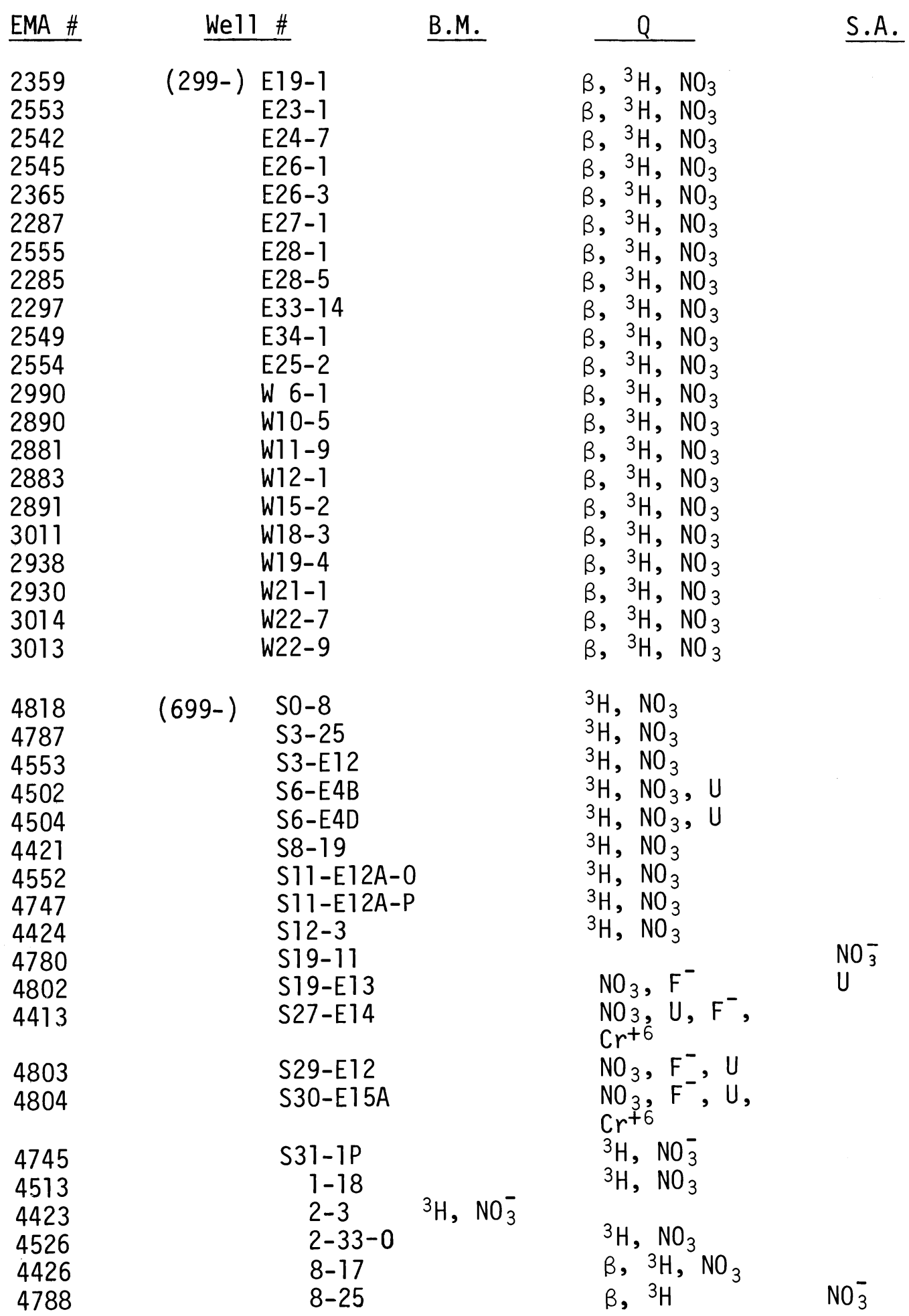


GROUNDWATER (continued)

Analyses

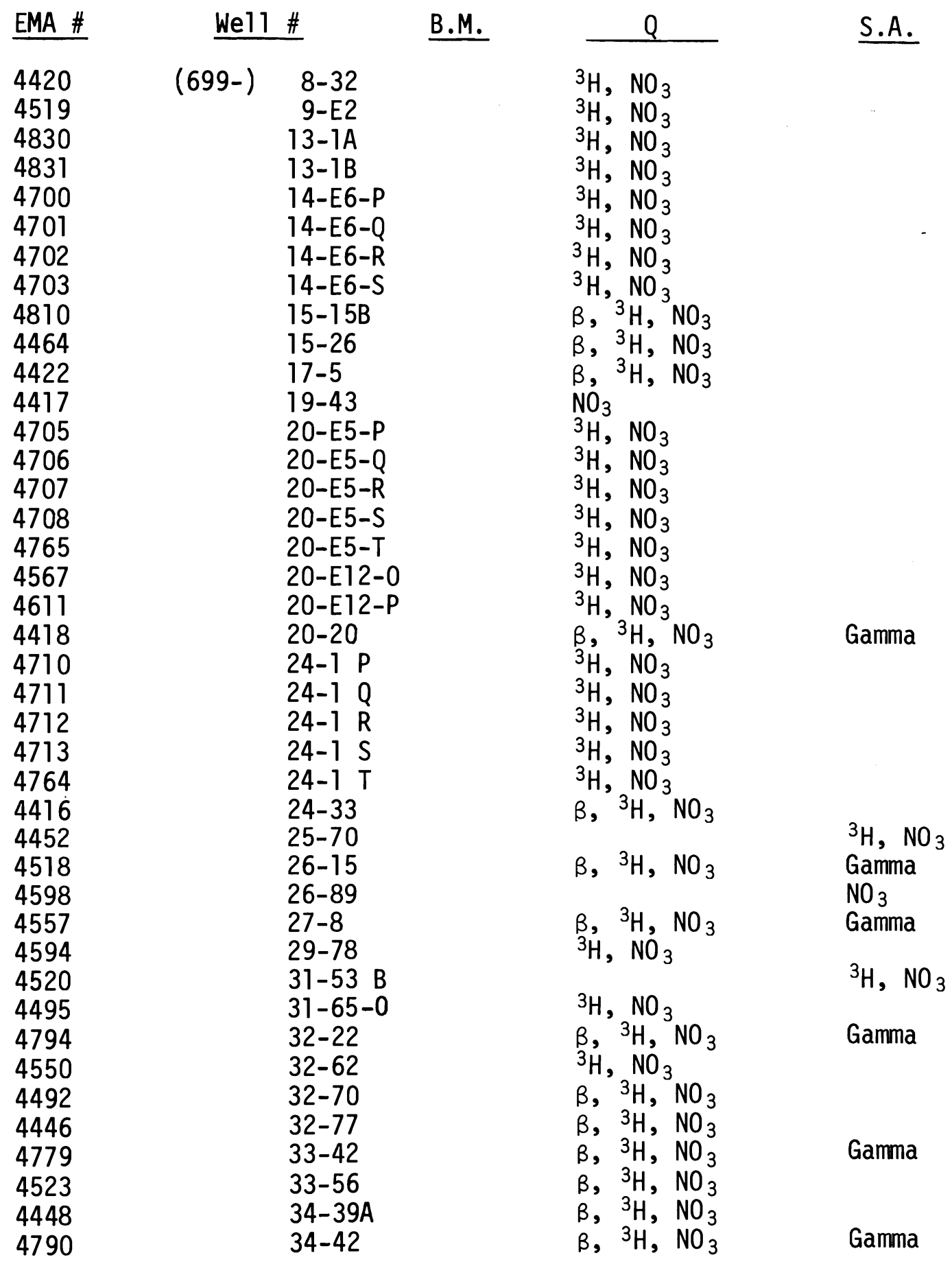


GROUNDWATER (continued)

Analyses

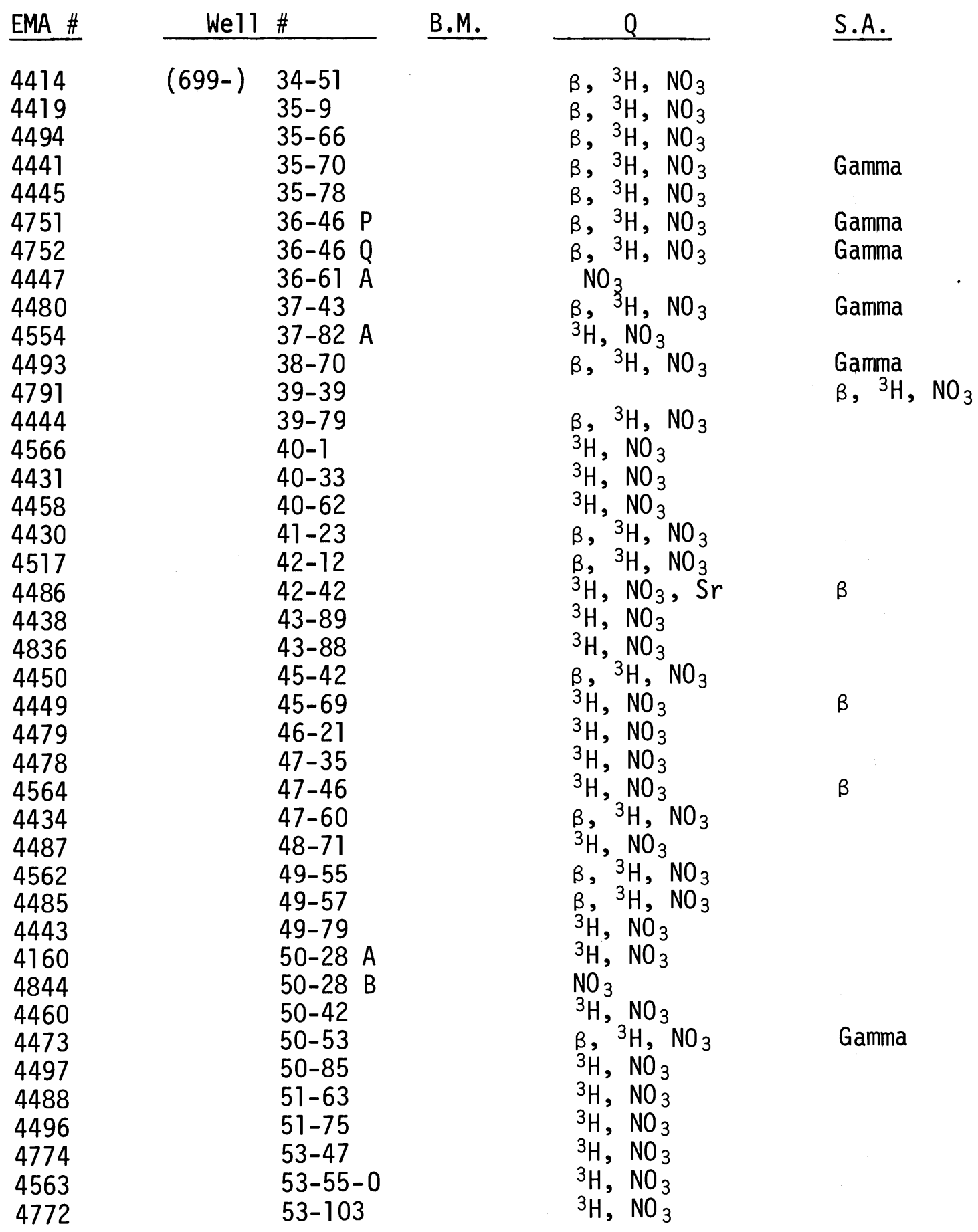


GROUNDWATER (continued)

Analyses

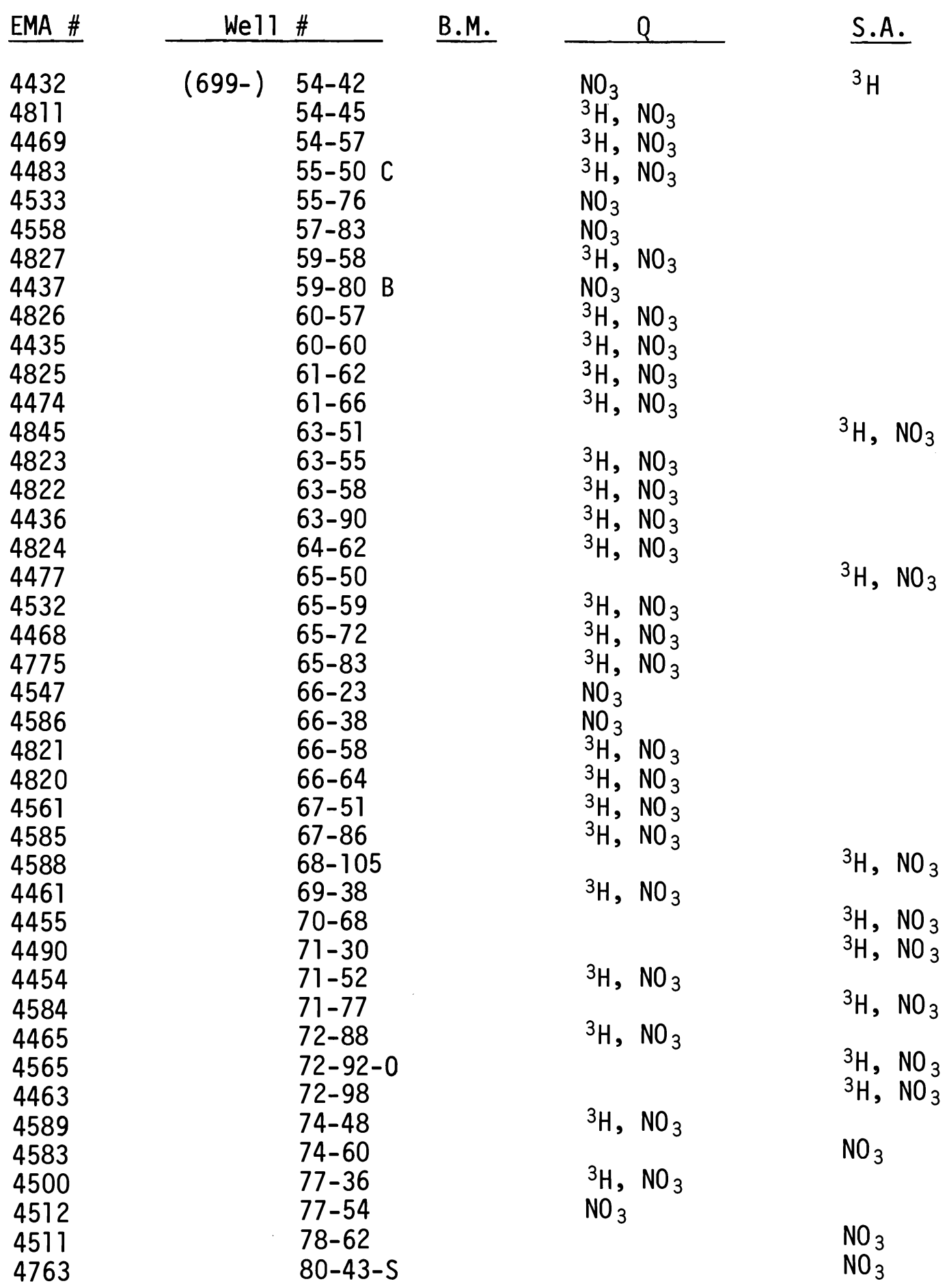


GROUNDWATER (continued)

Analyses

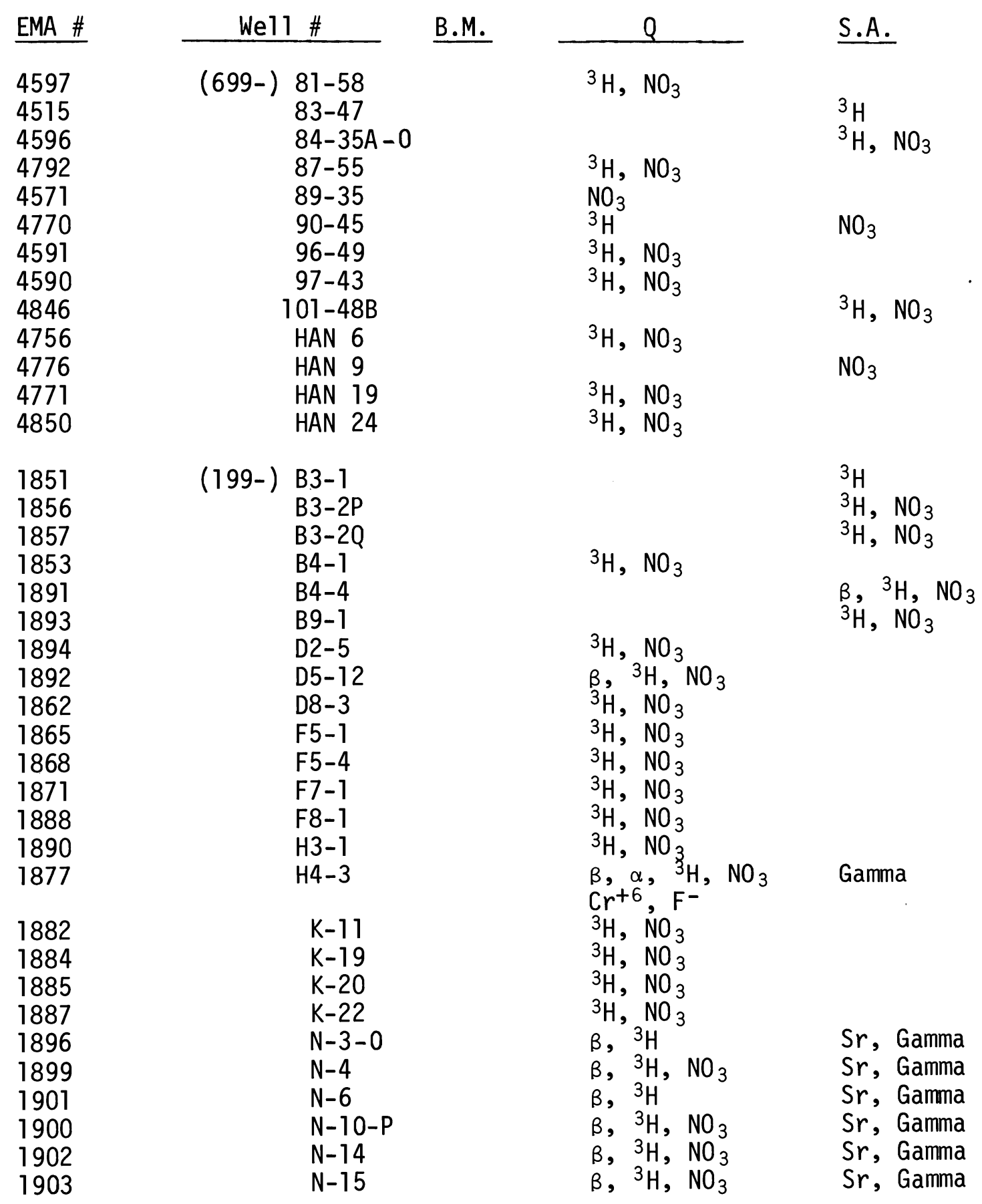


GROUNDWATER (continued)

Analyses

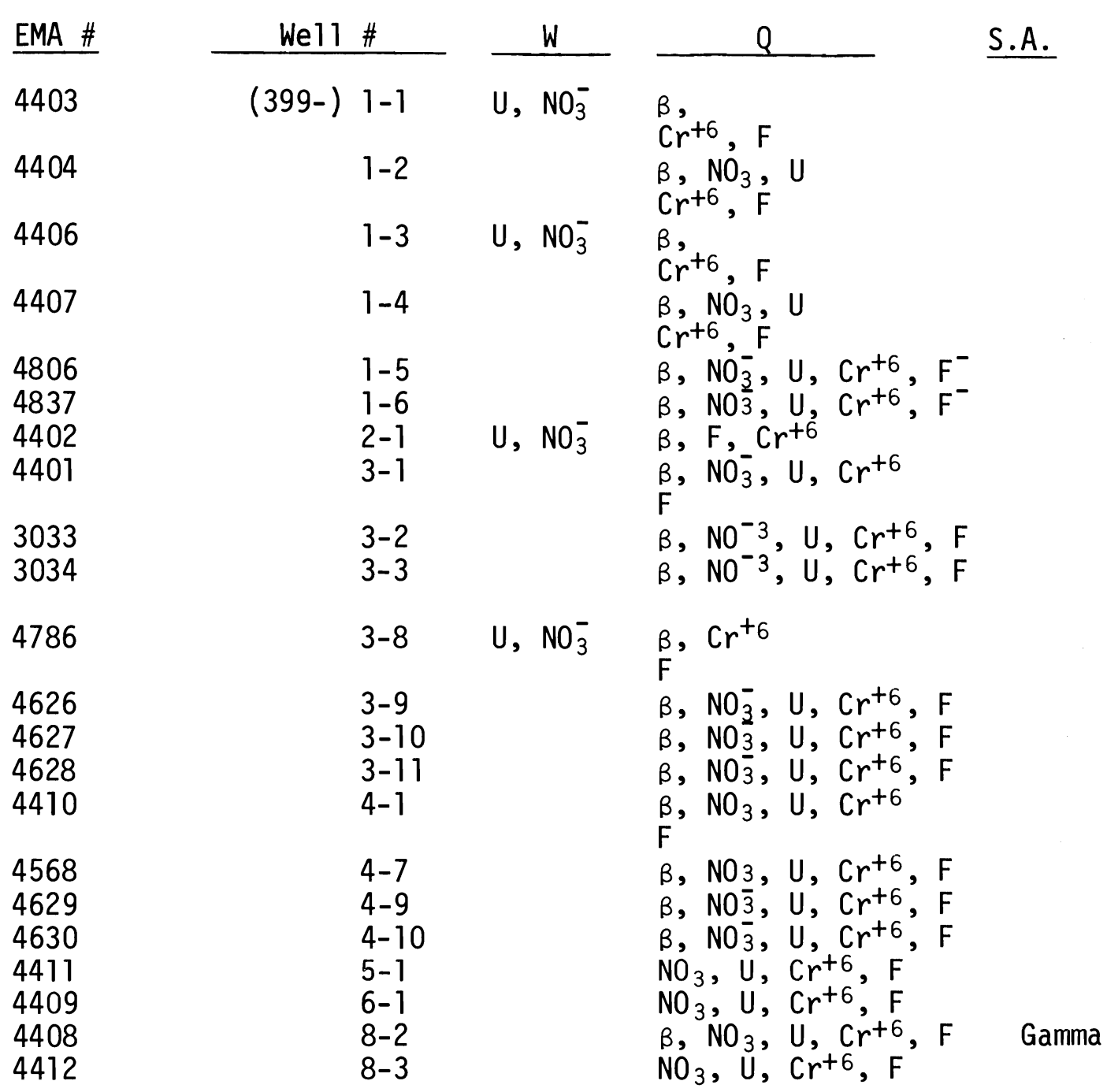

Quality of Water Analyses Annually

\begin{tabular}{|c|c|c|c|c|c|}
\hline (199-) & H4-3 & (699-) & $26-15$ & (699-) & $50-53$ \\
\hline (699-) & $\begin{array}{c}\text { S31-1P } \\
\text { S19-E13 } \\
\text { S3-E12 } \\
\text { S3-25 } \\
2-3 \\
10-54 \\
15-26 \\
19-43 \\
20-E 5 T \\
25-70\end{array}$ & & $\begin{array}{l}26-89 \\
31-53 B \\
34-42 \\
35-70 \\
36-93 \\
40-1 \\
40-33 \\
45-42 \\
45-69 \\
49-79 \\
50-28 B\end{array}$ & & $\begin{array}{l}55-50 C \\
59-58 \\
63-90 \\
66-64 \\
71-77 \\
72-88 \\
74-48 \\
78-62 \\
87-55 \\
89-35 \\
\text { HAN19 }\end{array}$ \\
\hline
\end{tabular}


VI. FOODSTUFFS

\begin{tabular}{|c|c|c|c|c|}
\hline Location & Description & EMA \# & Frequency & Analyses \\
\hline & & $\operatorname{MILK}^{(a)}$ & & \\
\hline Commercial & $\begin{array}{l}\text { Lucerne } \\
\text { Western Watershed }\end{array}$ & 0309 & $\begin{array}{l}M \\
Q\end{array}$ & $\begin{array}{l}131 \mathrm{I}, \text { gamma scan } \\
{ }^{89} \mathrm{Sr},{ }^{90} \mathrm{Sr}, \mathrm{Ca}\end{array}$ \\
\hline Commercial & $\begin{array}{l}\text { Darigold-Arden } \\
\text { Eastern Watershed }\end{array}$ & 1647 & $\begin{array}{l}M \\
Q\end{array}$ & $\begin{array}{l}131 \mathrm{I} \text {, gamma scan } \\
{ }^{90} \mathrm{Sr}, \mathrm{Ca}\end{array}$ \\
\hline Lazy Dee & Wahluke Slope & 6165 & $\begin{array}{l}\text { BW } \\
Q\end{array}$ & $\begin{array}{l}131 \mathrm{I} \text {, gamma scan } \\
{ }^{90} \mathrm{Sr}, \mathrm{Ca} .\end{array}$ \\
\hline Harris & Riverview & 0975 & $\begin{array}{l}\text { BW } \\
Q\end{array}$ & $\begin{array}{l}131 \mathrm{I}, \text { gamma scan } \\
{ }^{89} \mathrm{Sr},{ }^{90} \mathrm{Sr}, \mathrm{Ca}\end{array}$ \\
\hline Truhlicka & Benton City & 6296 & $\begin{array}{l}\text { BW } \\
Q\end{array}$ & $\begin{array}{l}131 \mathrm{I} \text {, gamma scan } \\
90 \mathrm{Sr}, \mathrm{Ca}\end{array}$ \\
\hline Pettett & Byers Landing & 6340 & $\begin{array}{l}B W \\
Q\end{array}$ & $\begin{array}{l}131 \mathrm{I} \text {, gamma scan } \\
{ }^{90} \mathrm{Sr}, \mathrm{Ca}\end{array}$ \\
\hline $\begin{array}{l}\text { Columbia Basin } \\
\text { Composite \#3 }\end{array}$ & $\begin{array}{l}\text { Taylor } \\
\text { Cooke Bros. } \\
\text { Price } \\
\text { Bleazard }\end{array}$ & 1648 & $\begin{array}{l}\text { BW } \\
Q\end{array}$ & $\begin{array}{l}131 \mathrm{I} \text {, gamma scan } \\
90 \mathrm{Sr}, \mathrm{Ca}\end{array}$ \\
\hline
\end{tabular}

MEAT

$\begin{array}{lllll}\text { Harris } & \text { Riverview } & 1292 & \text { SA } & \text { Gamma scan, }{ }^{90} \text { Sr } \\ \begin{array}{c}\text { Pasco Meat } \\ \text { Packers }\end{array} & \text { Commercial } & 1375 & Q & \text { Gamma scan, }{ }^{90} \text { Sr }\end{array}$

CHICKEN

$\begin{array}{lllll}\text { Harris (b) } & \text { Riverview } & 1193 & Q & \text { Gamma scan, }{ }^{90} \mathrm{Sr} \\ 01 \text { (b) }^{(b)} & \text { Riverview } & 6198 & Q & \text { Gamma scan, }{ }^{90} \mathrm{Sr} \\ \text { Safeway } & \text { Commercial } & 1157 & \text { SA } & \text { Gamma scan, }{ }^{90} \mathrm{Sr}\end{array}$

\footnotetext{
(a) Sample of forage also collected but not analyzed unless a positive result for ${ }^{31} 1$ is observed.

(b) Sample collected from one source only each quarter.
} 
VI. FOODSTUFFS (continued)

Location

Description

EMA \#

Frequency

Analyses

PRODUCE (leafy vegetables) $^{(a)}$

Harris

Riverview

1609

M

Gamma scan, ${ }^{90} \mathrm{Sr}$

Local Farm

Benton City

1612

BM

Gamma scan, ${ }^{90} \mathrm{Sr}$

Commercial

Tri-City

1601

M

Gamma scan, ${ }^{90} \mathrm{Sr}$

$\underline{E G G S}$

Harris (b)

Riverview

1191

$01 \operatorname{sen}^{(b)}$

Riverview

1756

1189

$M$

Gamma scan

${ }^{90} \mathrm{Sr}$

Gamma scan

$M$

${ }^{90} \mathrm{Sr}$

Safeway

Commercial

SA

Gamma scan

${ }^{90} \mathrm{Sr}$

OYSTERS

Commercial

Willipa Bay

0323

Q

Gamma scan $\left(Z n^{65}\right)$

(a) Sample collected only during growing season, May to October.

(b) Sample only from one source each month. 
VII. WILDLIFE

Location

Columbia River

100-K to

Richland

U Pond

Gable Pond

B Pond

T Pond

West Lake

300 Area Pond

100-K to Hanford

100-K to 100-D

\& 300 Area Vicinity

Varies
EMA \#

Frequency

Analyses

\section{FISH}

According to species

M

Gamma scan, ${ }^{90} \mathrm{Sr}$

DUCKS

Depends on species and

20/0ct-Dec

Gamma scan, ${ }^{90} \mathrm{Sr}$ location

According

to species

Q (4/year)

Q (4/year)

According

to species

According

to species

According

to species

According

to species

According

to species

GEESE

According

to species

Q (4/year)

Q (4/year)

Q (4/year)

Q (4/year)

Gamma scan, ${ }^{90} \mathrm{Sr}$ Liver - Total Pu

Gamma scan, ${ }^{90} \mathrm{Sr}$

Gamma scan, ${ }^{90} \mathrm{Sr}$

Gamma scan, ${ }^{90} \mathrm{Sr}$

Gamma scan, ${ }^{90} \mathrm{Sr}$ Liver - U

Gamma scan, ${ }^{90} \mathrm{Sr}$ Liver - Total Pu, U

\section{PHEASANTS}

According

to species

DEER

According to location
10/0ct-Dec

10/0ct-Dec

Gamma scan 
VII. WILDLIFE (continued)

Location

BC Crib Area

100-N Trench

Surface Ponds

Selected Waste

disposal sites
EMA \#

Frequency

\section{RABBITS}

6084

2 in May
2 in Nov.

Muscle - Gamma scan

Bone - ${ }^{90} \mathrm{Sr}$

Liver - Total Pu
$\underline{\operatorname{MICE}}^{(\mathrm{a})}$

1775

According

to species

and location
BM (Mar.-Sept.) Gamma scan, ${ }^{90} \mathrm{Sr}$

BM (Mar.-Sept.) Muscle - Gamma scan, ${ }^{90} \mathrm{Sr}$ Bone - ${ }^{90} \mathrm{Sr}$ Liver - Total Pu, (b) $u(b)$

\section{OTHER SMALL MAMMALS}

According

to species

As requested

Muscle - Gamma scan

Bone - ${ }^{90} \mathrm{Sr}$ and location

Liver - Total Pu, (b) $\mathrm{U}(\mathrm{b})$

\footnotetext{
(a) Up to three mice may be composited in a sample and analyzed as a whole.

(b) Total Pu for mammals from U Pond and BC Crib Area;

$U$ for mammals from U Pond, West Lake, and 300 Area Pond.
} 
VIII. SOIL AND VEGETATION

\section{Location}

Waluke \#2 Air Sampling Station Berg Ranch Air Sampling Station NE Corner of 100-N Area 100-F (control plot 58) Yakima Barricade Intersection Rts. 4 and $11 \mathrm{~A}$ (CP \#59) Hanford townsite (CP \#57)

SW of 200-W Area (CP \#60) $E$ of 200-W Area (CP \#60) 200-E Hill (CP \#61) E of Nuclear Eng. Burial Grounds Inter. of Rt. 4 and Army Loop Rd. Ringold 0.5 mile NE of FFTF SW of FFFF

E of ALE Field Laboratory Prosser Barricade $N$ of 300 Area Perimeter SE of 300 Area Perimeter (CP \#50) $\mathrm{NE}$ Corner of Exxon Site
EMA

Soil Vegetation Frequency Analyses

$6007 \quad 6053$

$6008 \quad 6054$

$6275 \quad 6290$

$6018 \quad 6064$

$6004 \quad 6050$

6065

6063

6066

6283

6068

6284

62.32

6055

6285

6286

6282

6278

6225

6279

6014

6280
6287

6227

6288

6060
A Gamma Scan, ${ }^{90} \mathrm{Sr}, \mathrm{U}, \mathrm{Pu}^{\star}$

A Gamma Scan, ${ }^{90} \mathrm{Sr}, \mathrm{U}, \mathrm{Pu}$

A Gamma Scan, ${ }^{90} \mathrm{Sr}, \mathrm{U}, \mathrm{Pu}$

A Gamma Scan, $90 \mathrm{Sr}, \mathrm{U}, \mathrm{Pu}$

A Gamma Scan, ${ }^{90} \mathrm{Sr}, \mathrm{U}, \mathrm{Pu}$

A Gamma Scan, $90 \mathrm{Sr}, \mathrm{U}, \mathrm{Pu}$

A Gamma Scan, $90 \mathrm{Sr}, \mathrm{U}, \mathrm{Pu}$

A Gamma Scan, ${ }^{90} \mathrm{Sr}, \mathrm{U}, \mathrm{Pu}$

A Gamma Scan, $90 \mathrm{Sr}, \mathrm{U}, \mathrm{Pu}$

A Gamma Scan, ${ }^{90} \mathrm{Sr}, \mathrm{U}, \mathrm{Pu}$

A Gamma Scan, $90 \mathrm{Sr}, \mathrm{U}, \mathrm{Pu}$

A Gamma Scan, $90 \mathrm{Sr}, \mathrm{U}, \mathrm{Pu}$

A Gamma Scan, $90 \mathrm{Sr}, \mathrm{U}, \mathrm{Pu}$

A Gamma Scan, $90 \mathrm{Sr}, \mathrm{U}, \mathrm{Pu}$

A Gamma Scan, $90 \mathrm{Sr}, \mathrm{U}, \mathrm{Pu}$

A Gamma Scan, $90 \mathrm{Sr}, U, \mathrm{Pu}$

A Gamma Scan, $90 \mathrm{Sr}, \mathrm{U}, \mathrm{Pu}$

A Gamma Scan, $90 \mathrm{Sr}, \mathrm{U}, \mathrm{Pu}$

A Gamma Scan, $90 \mathrm{Sr}, \mathrm{U}, \mathrm{Pu}$

A Gamma Scan, $90 \mathrm{Sr}, \mathrm{U}, \mathrm{Pu}$

\footnotetext{
${ }^{\star}$ Isotopic Pu.
} 
IX. EXTERNAL RADIATION MEASUREMENT

Thermoluminescent Dosimeters

\begin{tabular}{|c|c|c|c|}
\hline Location & EMA \# & Frequency & Mea surement \\
\hline Coyote Rapids & 6135 & $M$ & Immersion Dose \\
\hline Below $100-N$ & 6104 & $M$ & Immersion Dose \\
\hline Hanford Powerline & 6137 & $M$ & Immersion Dose \\
\hline Richland Pumphouse & 1715 & $M$ & Immersion Dose \\
\hline$\star 200$ ENC & 1467 & $M$ & Ambient Dose \\
\hline$\star 200 \mathrm{ESE}$ & 1468 & $M$ & Ambient Dose \\
\hline *200 EWC & 1470 & $M$ & Ambient Dose \\
\hline *200 EEC & 1469 & $M$ & Ambient Dose \\
\hline *200 WEC & 1473 & $M$ & Ambient Dose \\
\hline$\star$ Redox & 1474 & $M$ & Ambient Dose \\
\hline *200 WWC & 1471 & $M$ & Ambient Dose \\
\hline$\star 200$ WNE & 1472 & $M$ & Ambient Dose \\
\hline *3705 Bldg. & 1486 & $M$ & Ambient Dose \\
\hline *ACRMS & 1698 & $M$ & Ambient Dose \\
\hline *300 Pond & 1699 & $M$ & Ambient Dose \\
\hline *300 SW Gate & 6163 & $M$ & Ambient Dose \\
\hline *300 South Gate & 6162 & $M$ & Ambient Dose \\
\hline 331 Bldg. & 1765 & $M$ & Ambient Dose \\
\hline *C.P. \#63 & 6170 & $M$ & Ambient Dose \\
\hline *C.P. \#64 & 6171 & $M$ & Ambient Dose \\
\hline C.P. \#65 & 1708 & $M$ & Ambient Dose \\
\hline C.P. \#66 & 6172 & $M$ & Ambient Dose \\
\hline C.P. \#67 & 6173 & $M$ & Ambient Dose \\
\hline Wahluke C.P. \#17 & 1700 & $M$ & Ambient Dose \\
\hline Wahluke C.P. \#18 & 1701 & $M$ & Ambient Dose \\
\hline Wahluke C.P. \#19 & 1702 & $M$ & Ambient Dose \\
\hline Wahluke C.P. \#20 & 1703 & $M$ & Ambient Dose \\
\hline Wahluke C.P. \#21 & 1704 & $M$ & Ambient Dose \\
\hline Wahl uke C.P. \#22 & 1705 & $M$ & Ambient Dose \\
\hline Wahluke C.P. \#23 & 1706 & M & Ambient Dose \\
\hline Wahluke C.P. \#24 & 1707 & M & Ambient Dose \\
\hline Wahluke C.P. \#46 & 1728 & $M$ & Ambient Dose \\
\hline$\star 100-K$ & 1475 & $\ddot{M}$ & Ambient Dose \\
\hline *WPPSS to $100-N$ & 1476 & $M$ & Ambient Dose \\
\hline$\star 100-D$ & 1477 & $M$ & Ambient Dose \\
\hline *100 Area Fire Station & 6164 & $M$ & Ambient Dose \\
\hline Rt $10 \mathrm{Mi} \cdot 1.6$ & 1710 & $M$ & Ambient Dose \\
\hline FFTF site & 1729 & $M$ & Ambient Dose \\
\hline FFTF North & 6177 & $M$ & Ambient Dose \\
\hline FFTF Southeast & 6178 & M & Ambient Dose \\
\hline *Prosser Barricade & 6176 & M & Ambient Dose \\
\hline *100 F & 1478 & $M$ & Ambient Dose \\
\hline *Hanford & 1480 & $M$ & Ambient Dose \\
\hline
\end{tabular}

*Located at Air Sampling Station 
IX. EXTERNAL RADIATION MEASUREMENT (continued)

Thermoluminescent Dosimeters

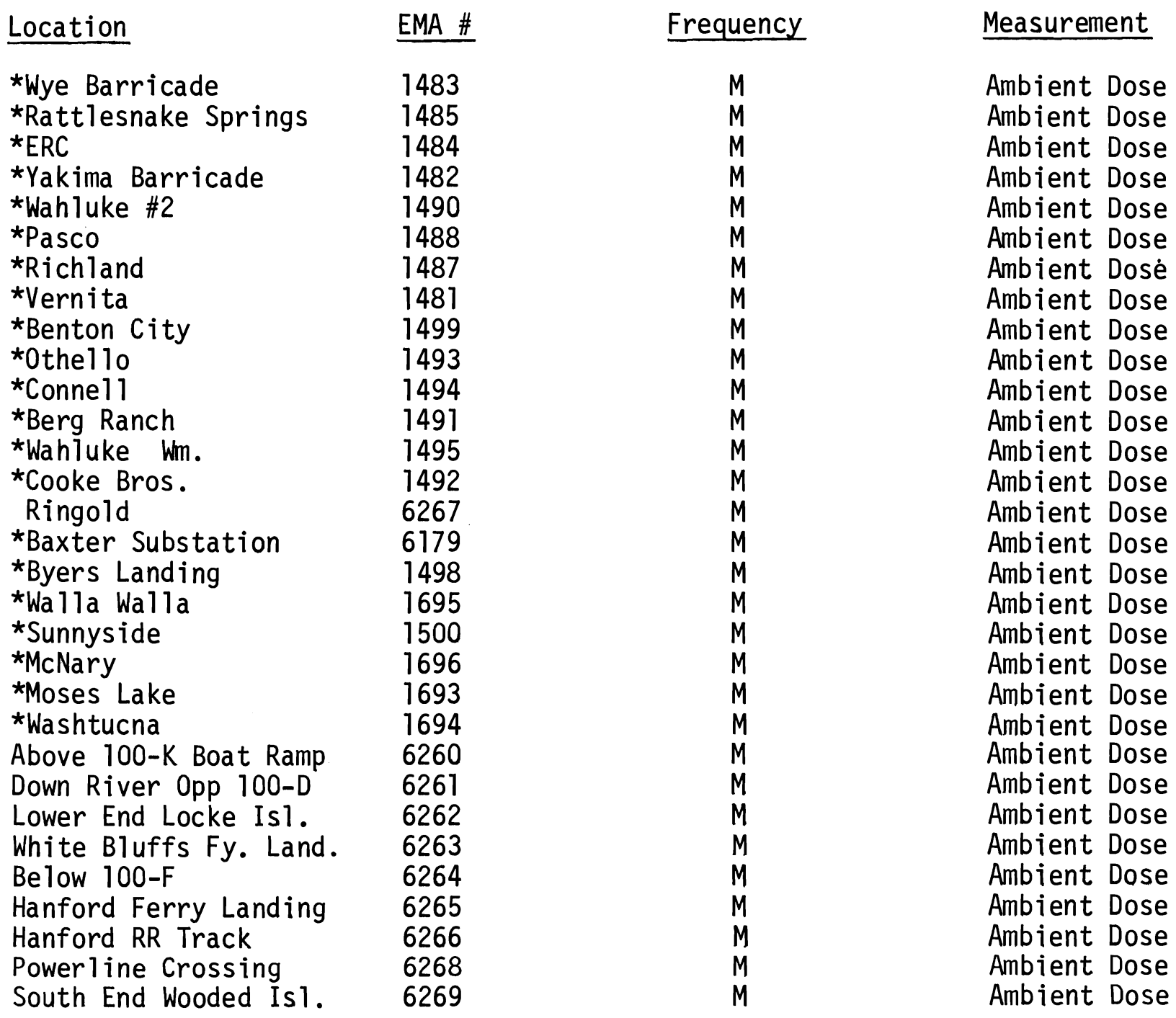

*Located at Air Sampling Station 
$X$. PORTABLE INSTRUMENT SURVEYS

Air Sampling Filters

$\begin{array}{llcl}\text { Location } & \text { Record } & \text { Frequency } & \text { Instrument } \\ 3705 \text { B Idg } & \text { Trip Sheet } & \text { D } & \text { GM, PAM } \\ \text { ACRMS } & \text { Trip Sheet } & \text { D } & \text { GM, PAM } \\ 300 \text { South Gate } & \text { Trip Sheet } & \text { D } & \text { GM, PAM } \\ 300 \text { Southwest Gate } & \text { Trip Sheet } & \text { D } & \text { GM, PAM }\end{array}$

Columbia River Shoreline

\begin{tabular}{|c|c|c|c|}
\hline Location & $\begin{array}{l}\text { River } \\
\text { Mile } \\
\end{array}$ & EMA \# & Instrument \\
\hline Coyote Rapids & $382.7 \mathrm{P}$ & 6240 & LLM, GM \\
\hline $\begin{array}{l}\text { Upstream of } 100-K \\
N \text { KE Basin }\end{array}$ & $382.5 P$ & 6132 & LLM, GM (TLD) \\
\hline $\begin{array}{l}\text { N KE Basin } \\
\text { Opposite KE Basin }\end{array}$ & $381.5 \mathrm{P}$ & 6131 & LLM, GM \\
\hline N Trench Springs & $\begin{array}{l}381.0 \mathrm{~F} \\
3794 \mathrm{P}\end{array}$ & $\begin{array}{l}6130 \\
6129\end{array}$ & LLM, GM \\
\hline Downstream of $100-\mathrm{N}$ & $379.1 \mathrm{P}$ & 6209 & LLM, GM (TLD) \\
\hline $\begin{array}{l}\text { Downs tream of } 100-\mathrm{N} \\
\text { Above } 100-\mathrm{D}\end{array}$ & $378.6 \mathrm{P}$ & 6128 & LLM, GM \\
\hline $\begin{array}{l}\text { Above } 100-D \\
D \text { Island }\end{array}$ & $378.1 \mathrm{P}$ & 6238 & LLM, GM \\
\hline $\begin{array}{l}\text { D Island } \\
\text { Opposite } 100-D\end{array}$ & $\begin{array}{l}3 / 1.0 \mathrm{lF} \\
376.8 \mathrm{~F}\end{array}$ & 6241 & LLM, GM (TLD) \\
\hline $\begin{array}{l}\text { Is l adjacent Upper End } \\
\text { Locke Isl. }\end{array}$ & $373.3 \mathrm{P}$ & 6124 & LLM, GM \\
\hline Locke Island-lower end & 371.0 IP & 6123 & LLM, GM (TLD) \\
\hline $\begin{array}{l}\text { White Bluffs Ferry Landing } \\
100-F\end{array}$ & $369.7 \mathrm{P}$ & 6121 & LLM, GM (TLD) \\
\hline $\begin{array}{l}\text { 100-F } \\
\text { Hanford Powerline }\end{array}$ & $\begin{array}{l}368.3 \mathrm{P} \\
3626 \mathrm{D}\end{array}$ & 6120 & $\begin{array}{l}\text { LLM, GM (TLD) } \\
\text { LLM, GM }\end{array}$ \\
\hline $\begin{array}{l}\text { Hanford Powerl ine } \\
\text { Hanford Ferry Landing }\end{array}$ & $362.0 \mathrm{~F}$ & $\begin{array}{l}6118 \\
6117\end{array}$ & $\begin{array}{l}\text { LLM, GM (TLD) } \\
\text { LLM, GM (TLD }\end{array}$ \\
\hline Hanford Railroad Track & $361.4 \mathrm{P}$ & 6242 & LLM, GM (TLD) \\
\hline Ringold & & 6114 & LLM, GM (TLD) \\
\hline Powerline Crossing & $350.4 \mathrm{P}$ & 6113 & LLM, GM (TLD) \\
\hline Powerline Crossing & $350.4 \mathrm{~F}$ & 6112 & LLM, GM \\
\hline Byers Landing & $345.2 \mathrm{~F}$ & 6111 & LLM, GM \\
\hline Port of Benton & $343.3 \mathrm{P}$ & 61 & LLM, GM \\
\hline Richland Pumphol & 340.5 & & LLM, GM \\
\hline
\end{tabular}

Roadways

Description

300 Area to $200-W$

Rt. 4-S \& Rt. 3

200-W to 300 Area

Rt. 3 \& Rt. 4-S
Identification

Number

1

2
Frequency

M

M
Instrument

Bioplastic Crystal

Bioplastic Crystal 
Roadways (continued)

Description

Hanford Highway

Horn Rapids to Yakima

Barricade \& Rt. 6

Wye Barricade on

Rt. 2-S to Rt. 11-A,

Rt. 11-A to Yakima

Barricade

Jct. Rt. $11-\mathrm{A}$ and $2 \mathrm{~N}$, on $2 \mathrm{~N}$, past $100 \mathrm{~F}, \mathrm{H}, \mathrm{D}$

to Rt. $4 \mathrm{~N}$, Rt. $4 \mathrm{~N}$ to

Rt. 1, on Rt. 1 past

$100 \mathrm{~K}, \mathrm{C}$ to Rt. 6 , Rt. 6

to Jct. with Rt. 11-A

Jct. Rt. 11-A and 6, on Rt. 6 to Rt. 1 ,

Rt. 1 to Rt. $4 \mathrm{~N}$, on

Rt. $4 \mathrm{~N}$ to Rt. $2 \mathrm{~N}$,

on Rt. $2 \mathrm{~N}$ to Jct.

with Rt. 11-A

Jct. Rt. 2 \& Rt. 1 , White Bluffs cutoff, Rt. $4 \mathrm{~N}$ to $200-\mathrm{E}$

from Rt. $4 \mathrm{~N}$ to $\mathrm{C}$

200-E Perimeter

200-W Perimeter

300 Area on Rt. 10

to Prosser Barricade

\& Wye Barricade

\section{Identification}

Number

3

Q

Bioplastic Crystal

4

Q

Bioplastic Crystal

5

Q

Bioplastic Crystal

$5 A$

Q

Bioplastic Crystal

6

Q

Bioplastic Crystal

7

M

Bioplastic Crystal

8

M

Bioplastic Crystal

10

Q

Bioplastic Crystal 
Roadways (continued)

Description

North Richland

Jct. $4 \mathrm{~N}$ and 100-N Access

$\mathrm{Rd}$ to $\mathrm{N}$-Area, $\mathrm{N}$-Area to RR

Crossing, left on Cutoff to

Rt. $4 \mathrm{~N}$ and back, Access Rd

to $4 \mathrm{~N}, \mathrm{Rt}$. $4 \mathrm{~N}$ to Rt. 1 ,

Rt. 1 to K-Area Access Rd,

Access Rd to K-Area, K-Area

to Rt. 1, Rt. 1 to Rt. $4 \mathrm{~N}$,

Rt. $4 \mathrm{~N}$ to Jct. with $100-\mathrm{N}$

Jct. Rt. 11-A \& Area 213

Access Rd, on Rt. 11-A to

Jct. Rt 3, Return

Army Loop Rd.

300 Area

Railroad Survey

Description

Frequency

A11 plant tracks outside area fences

SA
Frequency Instrument

Bioplastic Crystal

BW Bioplastic Crystal
13

14

15

M

Bioplastic Crystal

Bioplastic Crystal

Aerial Survey

Instrument

Bioplastic Crystal

\begin{tabular}{|c|c|c|c|}
\hline Description & $\begin{array}{c}\text { Identification } \\
\text { Number } \\
\end{array}$ & Frequency & Instrument \\
\hline Project Perimeter & 3 & A & $\mathrm{NaI}$ Crystal \\
\hline $\begin{array}{l}\text { Richland - } \\
\text { Ellensburg - } \\
\text { Ritzville Triangle }\end{array}$ & 4 & A & NaI Crystal \\
\hline $\begin{array}{l}\text { Columbia River - } \\
\text { Vernita to McNary }\end{array}$ & 5 & A & NaI Crystal \\
\hline
\end{tabular}


XI. SURVEILLANCE OF WASTE DISPOSAL SITES

Active, inactive, and retired waste disposal sites require periodic monitoring to assure appropriate maintenance. The following sites require surveillance:

Description

100-N Crib
100-N Trench
100-N Burning Ground
100-K Trench
100-K Solid Waste B.G.
100-K Burning Pit
100-BC SE B.G. (105-C Solid Waste)
100-BC SW B.G. (105-B Solid Waste, N. Solid Waste)
100-BC Construction B.G.
100-BC B.G. East of 108-B
100-BC Irradiated Metal Storage Basin Waste
108-B Bal1 3x Burial Ground
108-B Crib
105-C Trench
105-B Trench
107 Basin Sludge Burial
105-C Metal Examination Waste Tank
100-BC Overflow Pluto Crib
107-C Retention Basin
107-B Retention Basin
100-BC Effluent Diversion Box
100-BC Minor B.G.'s East of 105-B
100-BC Outfal1 Structures
100-DR Outfall Structures
100-DDR Trench
107-D Retention Basin
107-DR Retention Basin
100-DDR Effluent Lines
100-D Dummy Decontamination Waste
100-DDR Solid Waste B.G. (VSR Thimbles)
100-DDR Construction B.G.
100-DDR \#3 B.G. NE of DR
100-DDR Pluto Crib
100-DDR 105 Basin Sludge B.G.
100-DDR \#1 B.G.
100-DDR \#2 B.G.
100-H Trench
107-H Basin
100-H Effluent Lines (Junction Boxes)
100-H Liquid Waste Burial
100-H \#1 B.G.
100-H \#2 B.G.

Frequency

SA

Q

$S A$

SA

$Q$

$S A$

$S A$

Q

$S A$

$S A$

$S A$

$S A$

$S A$

$S A$

$S A$

$S A$

$S A$

SA

SA

$S A$

$S A$

$S A$

SA

$S A$

$S A$

SA

$S A$

$S A$

$S A$

$S A$

SA

$S A$

$S A$

$S A$

$S A$

$S A$

SA

$S A$

$S A$

$S A$

$S A$

$S A$ 
XI. SURVEILLANCE OF WASTE DISPOSAL SITES (continued)

Description

100-F Area

P-11 Area

100-F Lewis Cana 1

100-F Swampy Area

107-F Trench

100-F Retention Basin

100-F Trench Drain and Adjacent Wood Covered Pit

100-F Ball Washer Crib

100-F \#3 B.G.

$100-F$ \#2 B.G.

$100-F$ \#1 B.G.

100-F Sawdust Burial

100-F Leaching Trench

100-F 60" Overground Pipe

100-F Happy Valley Farm Plots

200-W Redox Labs Pond (216-S-19) •

200-W New Redox Pond (216-S-16)

200-W 01d Redox Pond (216-S-17)

200-W Part of Perimeter of U Pond (216-U-10)

200-W U Pond Overflow (216-U-11)

200-E Gable Mt. Ponds (216-A-25)

200-E West Lake

200-E B Pond (216-B-3)

200-E B Pond Ditch \#1

200-E B Pond Ditch \#2

200-E B Pond Ditch \#3

200-E Snow's Canyon; Purex Chemical Sewer (216-A-29)

200-E Purex Crib \#1 (216-A-6)

200-E Purex Crib \#2 (216-A-30-1)

200-E North of Purex Crib \#3 (216-A-37-1)

200-E NE Perimeter Fence

200-E 216-BC Crib Area

200-E California Nuclear B.G. Perimeter

200-E - 200-W Transfer Lines

300 Area 300 N B.G.

300 Area 300 Wye B.G.

300 Area \#1 B.G.

300 Area \#2 B.G.

300 Area \#3 B.G.

300 Area \#4 B.G.

300 Area \#5 B.G.

300 Area \#7 B.G.

300 Area \#8 B.G.

300 West B.G.

300 Area Equipment Storage

300 Area N. Process Pond at Perimeter Fence

300 Area S. Process Pond at Perimeter Fence 200-N, P, and R Areas
Frequency

A

A

SA

SA

SA

$S A$

$S A$

$S A$

$S A$

SA

$Q$

$Q$

SA

SA

$Q$

SA

Q

0

$S A$

$Q$

SA

$Q$

Q

Q

$S A$

Q

$S A$

SA

Q

$S A$

SA

$S A$

$S A$

$S A$

$S A$

$S A$

$Q$

$S A$

$S A$

$S A$

$S A$

Q

Q

SA 
DISTRIBUTION

No. of

Copies

OFFSITE

1 A. A. Churm

ERDA Patent Group

U.S. Energy Research and Development Administration 9800 South Cass Avenue

Argonne, IL 60439

27 USERDA Technical Information Center

\section{ONSITE}

10 Energy Research and Development Administration

Richland Operations office

Environmental Affairs

0. J. Elgert

L. F. Perkins

P. G. Rhoades

F. R. Standerfer

M. W. Tiernan (6)

5 Atlantic Richfield Hanford Co.

G. L. Hanson

R. E. Isaacson

H. L. Maxfield

V. Panesko

ARHCO Files

1 Hanford Environmental Health Foundation

R. G. Anderson

3 United Nuclear Industries, Inc.

T. E. Dabrowski

A. E. Engler

UNI Files
No. of

Copies

$\underline{\text { ONSITE }}$

2 Westinghouse Hanford Co.

G. D. Carpenter

R. B. Hall

71 Battelle-Northwest

G. L. Andersen

P. L. Baker

P. J. Blumer (41)

P. E. Bramson

J. P. Corley

J. J. Fix

R. F. Foster

J. J. Jech

H. V. Larson

C. A. McCoy

V. L. McGhan

T. J. McLaughl in

M. L. Miller

D. G. Morton

D. A. Myers

M. R. Quarders

W. H. Rickard

R. C. Schrotke (5)

R. W. Simmons

J. Skinner

J. H. Soehnlein

J. K. Soldat

C. M. Unruh

J. A. Warder

Technical Information (2)

Technical Publications 
. 